\title{
A Method for Gas Identification in Thermal Dispersion Mass Flow Meters
}

\author{
Klemen Rupnik* - Jože Kutin - Ivan Bajsić \\ University of Ljubljana, Faculty of Mechanical Engineering, Slovenia
}

\begin{abstract}
A novel measurement method for identifying the type of gas in a thermal dispersion mass flow meter is presented. The physical background of the gas-identification method is discussed by employing a simple, one-dimensional, mathematical model of a thermal flow sensor. For a practical realization of the gas-identification method, the thermal dispersion mass flow meter has to contain two thermal flow sensors with different constructional or operational parameters. A thermal dispersion mass flow meter containing two thermal flow sensors with circular and square cross-sections was developed and calibrated for five different gases in order to experimentally validate the gas-identification method. If the measurement characteristics for the improper gas are employed, the mass flow readings of the thermal flow sensors will generally differ. The absolute value of the relative difference in the mass flow readings can be used as an objective function for identifying the type of gas from a defined set of gases with known compositions. In addition, the normalized error is proposed as an objective function to consider the dispersion that could be reasonably attributed to the difference in the mass flow readings.
\end{abstract}

Keywords: gas-identification method, thermal dispersion mass flow meter, thermal flow sensors, different constructional parameters, measurement characteristics, experimental validation

\section{INTRODUCTION}

The measuring principle of a thermal mass flow meter is based on the influence of fluid flow (in most cases, a gas flow) on the heat transfer from a heated element [1] and [2]. The thermal mass flow meters are of two basic types: capillary thermal mass flow meters and thermal dispersion mass flow meters. In capillary thermal mass flow meters, the mass flow induces an asymmetry of the temperature profile along the heated capillary tube. In thermal dispersion mass flow meters (both the insertion and the in-line types), the gas is flowing around a thermal flow sensor. The thermal flow sensor typically contains a resistance temperature sensing element that is heated by the supplied electrical power, which results in the increased temperature of the sensing element. A constant temperature difference between the thermal flow sensor and the gas is usually maintained, and the required electrical heating power changes with the mass flow rate. Another possibility is that the thermal dispersion mass flow meter operates with a constant electrical heating power and the established temperature difference changes with the mass flow rate. The operating principles, construction and applications of industrial-grade thermal dispersion mass flow meters were presented by Olin [3] and [4].

The performance of thermal dispersion mass flow meters is affected by the internal structure of the thermal flow sensor, the installation conditions and the process conditions. Badarlis et al. [5] performed the optimization of the heater position within a thermal flow sensor and proposed a new heater design. Baker and Gimson [6] investigated the influence of the eccentricity of the sensor's internal structure, and the effects of the insertion length of the sensor and the construction details at the location where the sensor is inserted into the flow pipe. The effect of misaligned flow pipes and the influence of a single bend upstream of the thermal flow sensor were analysed by Gibson [7]. Pape et al. [8] developed a method for correcting the effect of the coatings that are deposited on the thermal flow sensor, which can be carried out simultaneously with a measurement of the mass flow rate. Pape and Hencken [9] investigated a coating diagnostics method that is realized with the help of an additional temperature sensing element within the thermal flow sensor.

Besides the mass flow rate (or the local mass velocity) of a gas, the intensity of the convective heat transfer from the thermal flow sensor to the gas is also affected by the thermodynamic and transport properties of the gas, such as the thermal conductivity, the specific heat at constant pressure and the dynamic viscosity. Therefore, the measurement characteristic of a thermal dispersion mass flow meter depends on both the composition and the type of the gas. If a thermal dispersion mass flow meter is employed to measure the mass flow rate of a gas that is different to the gas used for the calibration, an appropriate correction needs to be applied. Such corrections were investigated by, e.g., Lötters [10], Hardy et al. [11] and Popp [12]. It is also possible to perform the calibration for a variety of gases and then the proper measurement characteristic should be selected when the thermal dispersion mass flow meter is being used. 
In both solutions, the gas that is actually flowing through the thermal mass flow meter has to be known. In contrast, the method presented in this paper is capable of an in-situ identification of the type of gas from a defined set of gases with known compositions. The gas-identification method is realized by means of a thermal dispersion mass flow meter containing two different thermal flow sensors. The thermal dispersion mass flow meter with the capability to perform the gas-identification method and the gas-identification method itself are patent pending [13].

This paper is structured as follows. The physical background of the gas-identification method is given in Section 1. In Section 2 the experimental validation of the gas-identification method is presented. Section 3 comprises conclusions based on the findings, and open questions for further research work.

\section{PHYSICAL BACKGROUND}

A thermal dispersion mass flow meter containing two different thermal flow sensors ( 1 and 2$)$ is considered. The output signals of the thermal flow sensors can be written as $(P / \Delta T)_{1}$ and $(P / \Delta T)_{2}$, where $P$ is the supplied electrical heating power and $\Delta T$ is the maintained temperature difference between the thermal flow sensor and the gas. The measurement characteristics of the thermal flow sensors relate the corresponding output signals and the measured mass flow rate $\dot{m}$.



Fig. 1. Measurement characteristics of two different thermal flow sensors ( 1 and 2) for two different gases ( $A$ and $B$ ) and the basic principle of the gas-identification method

Fig. 1 shows the basic principle of the gasidentification method. The curves represent the measurement characteristics of two different thermal flow sensors (1 and 2) for two different gases ( $A$ and $B$ ). Gas $A$ is considered to be the actual gas flowing through the thermal dispersion mass flow meter. If the proper measurement characteristics (in this case for gas $A$ ) are employed, the mass flow readings of both thermal flow sensors will be equal, $\dot{m}_{1}^{(A)}=\dot{m}_{2}^{(A)}$. In contrast, if the improper measurement characteristics (in this case for gas $B$ ) are employed, the mass flow readings will differ, $\dot{m}_{1}^{(B)} \neq \dot{m}_{2}^{(B)}$.

A simple, one-dimensional, mathematical model will be employed to study the basic parameters that influence the results of the gas-identification method. The thermal flow sensor typically comprises a (heated) sensing element on a support structure, a filler material and a sheath, which is schematically presented in Fig. 2.

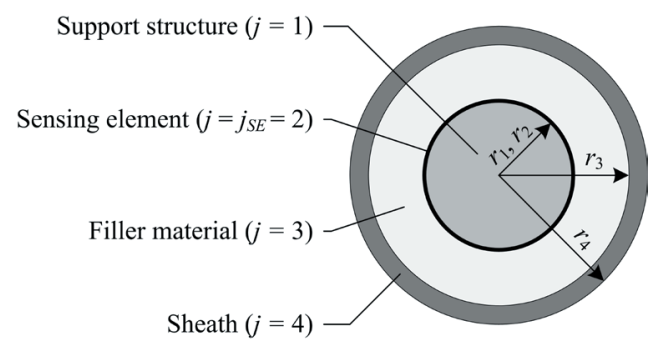

Fig. 2. Scheme of the cross-sectional view of a thermal flow sensor

The thermal flow sensor is assumed to be inserted into a pipe with a uniform gas flow with the temperature $T_{g}$ and the mass flow rate $\dot{m}=\rho \bar{V} A_{p}$, where $\rho$ is the density of the gas, $\bar{V}$ is the average velocity and $A_{p}$ is the pipe cross-sectional area. The intensity of the convective heat transfer from the surface of the thermal flow sensor to the gas flow is characterized by the convective heat transfer coefficient $h$. The temperature of the sensing element (represented as the layer $j_{S E}$ in Fig. 2) is $T=T_{g}+\Delta T$. A constant temperature difference $\Delta T$ is maintained by supplying electrical heating power $P=R I^{2}$ to the sensing element, where $R$ is the electrical resistance of the sensing element and $I$ is the electrical current passing through it. Assuming a one-dimensional heat transfer only in the radial direction, the output signal of the thermal flow sensor can be written as [14]:

$$
\frac{P}{\Delta T}=\frac{1}{\sum_{j=j_{S E}+1}^{N} \frac{\ln \left(r_{j} / r_{j-1}\right)}{2 \pi L \lambda_{j}}+\frac{1}{2 \pi r_{N} L h}},
$$

where $r_{j}$ and $\lambda_{j}$ are the outer radius and the thermal conductivity of the $j^{\text {th }}$ layer in the thermal flow sensor, respectively, $N$ is the number of all the layers and $L$ is the length of the sensing element. The presented mathematical model neglects the conductive heat transfer in the axial direction and the radiation heat transfer, and assumes a concentric internal structure 
of the thermal flow sensor and the constant thermal conductivities of the layers [15].

The convective heat transfer for a cylinder in a cross-flow is often characterized by a power model [14]:

$$
\mathrm{Nu}=a \operatorname{Pr}^{m} \operatorname{Re}^{n},
$$

which relates the Nusselt number $\mathrm{Nu}=h D / \lambda$, the Prandtl number $\operatorname{Pr}=c_{p} \eta / \lambda$ and the Reynolds number $\operatorname{Re}=\rho \bar{V} D / \eta$, where $D$ is the external characteristic length of the thermal flow sensor, and $a, m$ and $n$ are the parameters of the power model, which are generally dependent on the sensor geometry and the Reynolds number. The thermal conductivity $\lambda$, the specific heat at constant pressure $c_{p}$ and the dynamic viscosity $\eta$ of the gas are evaluated at the film temperature:

$$
T_{f}=\left(T_{s}+T_{g}\right) / 2,
$$

where $T_{S}$ is the temperature of the sensor surface. Considering $\bar{V}=\dot{m} / \rho A_{p}$, the convective heat transfer coefficient can be written as:

$$
h=a D^{n-1} A_{p}^{-n} c_{p}^{m} \lambda^{1-m} \eta^{m-n} \dot{m}^{n} .
$$

It is evident that the properties of the gas and the mass flow rate affect the output signal of the thermal flow sensor through the convective heat transfer coefficient. If the dimensions and the thermal properties of the sensor are considered to be constant, the output signal can generally be presented as $P / \Delta T=P / \Delta T(h)$ for a particular thermal flow sensor (valid for a circular shape, as in Eq. (1), or other shape of the cross-section).

Let us consider that the output signal of the $i^{\text {th }}$ thermal flow sensor is $(P / \Delta T)_{i}$. As evident from Fig. 1, there is a difference between the mass flow readings $\dot{m}_{i}^{(A)}$ and $\dot{m}_{i}^{(B)}$, which are calculated from the measurement characteristics for the gases $A$ and $B$, respectively. This difference between the mass flow readings can be evaluated from the equality $(P / \Delta T)_{i}^{(A)}=(P / \Delta T)_{i}^{(B)}$, which is fulfilled by the equal heat transfer coefficients:

$$
h_{i}^{(A)}=h_{i}^{(B)} .
$$

Considering Eqs. (4) and (5), the ratio between the mass flow readings of the $i^{\text {th }}$ thermal flow sensor is:

$$
\frac{\dot{m}_{i}^{(B)}}{\dot{m}_{i}^{(A)}}=\left(\frac{c_{p, i}^{(A)}}{c_{p, i}^{(B)}}\right)^{\frac{m_{i}}{n_{i}}}\left(\frac{\lambda_{i}^{(A)}}{\lambda_{i}^{(B)}}\right)^{\frac{1}{n_{i}}-\frac{m_{i}}{n_{i}}}\left(\frac{\eta_{i}^{(A)}}{\eta_{i}^{(B)}}\right)^{\frac{m_{i}}{n_{i}}-1} .
$$

To perform the gas-identification method, the mass flow readings of both thermal flow sensors should be obtained. If the actual gas is gas $A$ and the measurement characteristics for gas $A$ are employed, the mass flow readings of both thermal flow sensors are (theoretically) equal:

$$
\dot{m}_{1}^{(A)}=\dot{m}_{2}^{(A)} .
$$

In contrast, if the actual gas is gas $A$ and the measurement characteristics for gas $B$ are employed, the mass flow readings of the thermal flow sensors are not equal. The relative difference in the mass flow readings, defined as:

$$
\varepsilon=\frac{\dot{m}_{2}^{(B)}}{\dot{m}_{1}^{(B)}}-1,
$$

can be derived from Eqs. (6) and (7):

$$
\varepsilon=\frac{\left(\frac{c_{p, 2}^{(A)}}{c_{p, 2}^{(B)}}\right)^{\frac{m_{2}}{n_{2}}}\left(\frac{\lambda_{2}^{(A)}}{\lambda_{2}^{(B)}}\right)^{\frac{1}{n_{2}}-\frac{m_{2}}{n_{2}}}\left(\frac{\eta_{2}^{(A)}}{\eta_{2}^{(B)}}\right)^{\frac{m_{2}}{n_{2}}-1}}{\left(\frac{c_{p, 1}^{(A)}}{c_{p, 1}^{(B)}}\right)^{\frac{m_{1}}{n_{1}}}\left(\frac{\lambda_{1}^{(A)}}{\lambda_{1}^{(B)}}\right)^{\frac{1}{n_{1}}} \frac{m_{1}}{n_{1}}\left(\frac{\eta_{1}^{(A)}}{\eta_{1}^{(B)}}\right)^{\frac{m_{1}}{n_{1}}-1}}-1 .
$$

If the surface temperatures for both thermal flow sensors are equal, $T_{s, 1}=T_{s, 2}$, the corresponding gas properties are also equal, $c_{p, 1}=c_{p, 2}, \lambda_{1}=\lambda_{2}$ and $\eta_{1}=\eta_{2}$. Because $m$ is typically a constant value of about $1 / 3$ and is independent of the sensor geometry and the Reynolds number [3] and [14], $m_{1}=m_{2}$ is also taken into account, and Eq. (9) simplifies to:

$$
\varepsilon=\left[\left(\frac{c_{p}^{(A)}}{c_{p}^{(B)}}\right)^{m}\left(\frac{\lambda^{(A)}}{\lambda^{(B)}}\right)^{1-m}\left(\frac{\eta^{(A)}}{\eta^{(B)}}\right)^{m}\right]^{\frac{1}{n_{2}}-\frac{1}{n_{1}}}-1,
$$

or written in the form with the Prandtl number:

$$
\varepsilon=\left[\frac{\lambda^{(A)}}{\lambda^{(B)}}\left(\frac{\operatorname{Pr}^{(A)}}{\operatorname{Pr}^{(B)}}\right)^{m}\right]^{\frac{1}{n_{2}}-\frac{1}{n_{1}}}-1 .
$$

Fig. 3 presents the relative differences in the mass flow readings for different gases as a function of the parameter $n_{2}$, where the parameter $n_{1}$ is set to a constant value of 0.5 and air is considered to be the actual gas (gas $A$ ). The thermodynamic and transport properties of the gases were determined using the NIST REFPROP database [16] for a film temperature of $25{ }^{\circ} \mathrm{C}$ and a pressure of $100 \mathrm{kPa}$. If the proper 
measurement characteristics are employed (in this case for air), then $\varepsilon=0$. In contrast, if the measurement characteristics for oxygen, nitrous oxide, carbon dioxide or argon are employed, $|\varepsilon|>0$ and $|\varepsilon|$ increases with the difference between $n_{2}$ and $n_{1}$.

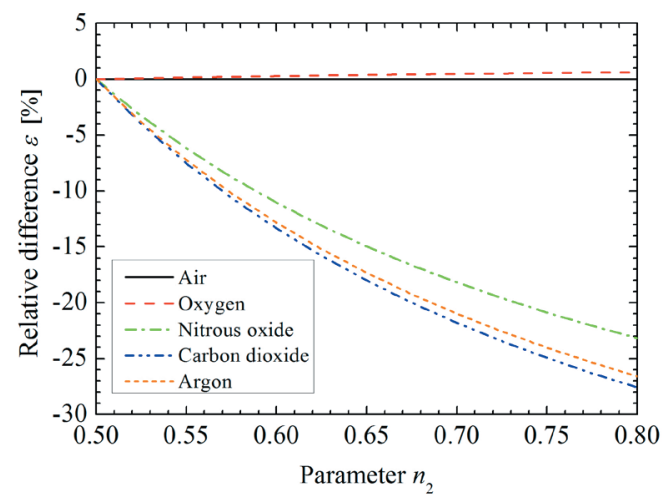

Fig. 3. Relative differences in the mass flow readings for different gases as a function of the parameter $n_{2}$, for $n_{1}=0.5$ and air as the actual gas

For a practical realization of the gas-identification method (as presented in Section 2), the relative difference in the mass flow readings should be as large as possible, if the improper measurement characteristics are employed. It is evident from Eqs. (9) to (11) and Fig. 3 that the relative difference in the mass flow readings can be influenced by the following parameters:

- The value of the parameter $n$ generally depends on the Reynolds number and the sensor geometry [14] and [17]. Different values of the parameters $n_{1}$ and $n_{2}$ can therefore be achieved by different constructional parameters of the thermal flow sensors, e.g., by different shapes, orientations with respect to the flow direction or characteristic lengths (which results in different Reynolds numbers at a given mass flow rate). Some examples of the parameter $n$ for different sensor geometries, for Reynolds numbers of the order of 10,000 [14], are presented in Fig. 4. At a given mass flow rate, similar Reynolds numbers are conditional on similar characteristic lengths of the sensors. The relative difference in the mass flow readings $\varepsilon$ depends on the difference between $n_{2}$ and $n_{1}$ (as shown in Fig. 3) and therefore an appropriate combination of the sensors should be selected to obtain a sufficiently large value of $\varepsilon$.

- The thermodynamic and transport properties of the gas are referred to the film temperature (see Eq. (3)) and so they are influenced by the maintained temperature of the sensing element.
A different influence of the gas properties can therefore be achieved by different operational parameters of the thermal flow sensors, e.g., by maintaining different temperatures of the sensing elements.
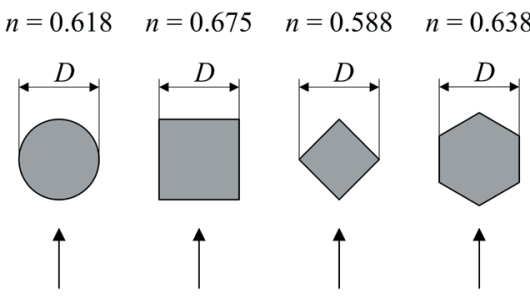

Flow direction

Fig. 4. Values of the parameter $n$ for sensors with different shapes of cross-sections or orientations with respect to the flow direction, for Reynolds numbers of the order of 10,000 [14]

\section{EXPERIMENTAL VALIDATION}

A thermal dispersion mass flow meter containing two thermal flow sensors with different constructional parameters was developed. It was calibrated for five different gases and used for the experimental validation of the gas-identification method.

\subsection{Measurement System}

The measurement system, employed for both the calibration of the developed thermal dispersion mass flow meter and the experimental validation of the gas-identification method, is schematically presented in Fig. 5. Besides the thermal dispersion mass flow meter, it comprises a gas source (GS), heat exchangers (HE1 to HE3), pressure regulators (PR1 and PR2) and a reference mass flow measurement system (REF). The role of the heat exchangers is to provide a stable gas temperature for the reference mass flow measurement system and for the thermal dispersion mass flow meter. The pressure regulators are used to set a stable inlet pressure for the reference mass flow measurement system with critical flow Venturi nozzles (TetraTec Instruments) [18], where the reference mass flow rate is generated and measured. The expanded measurement uncertainty of the reference mass flow rate does not exceed $0.3 \%$. The expanded measurement uncertainty characterizes the dispersion of the values that could reasonably be attributed to the measurand within an interval having a level of confidence of approximately $95 \%$ (coverage factor $k=2$ ) [19].

The developed thermal dispersion mass flow meter contains two heated Pt100 resistance 


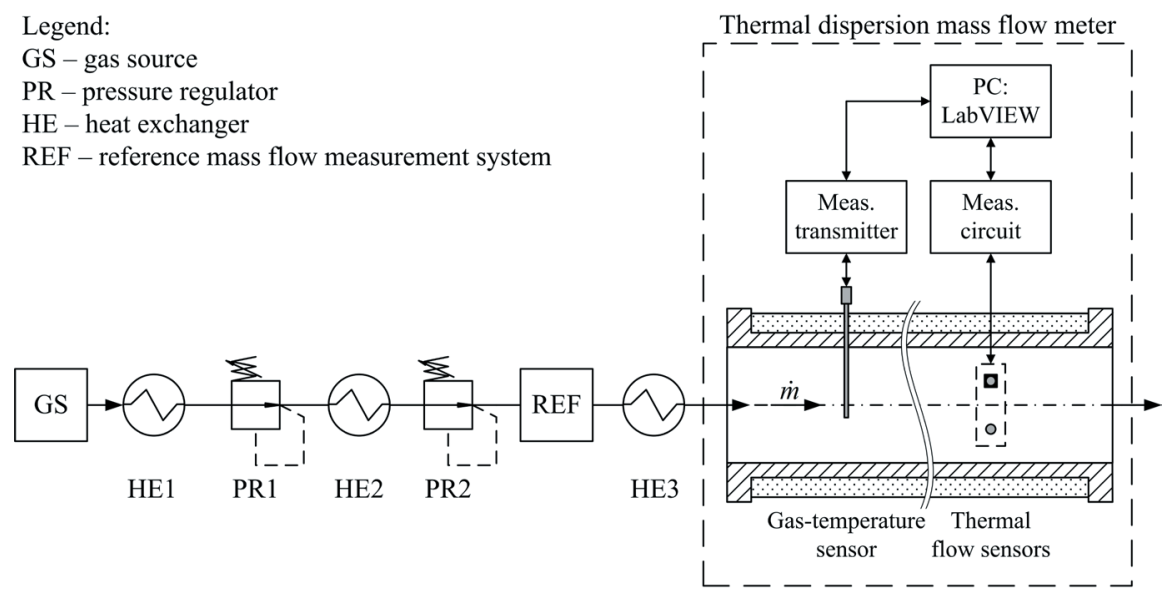

Fig. 5. Scheme of the measurement system

temperature sensors (TetraTec Instruments) that operate in the functions of the thermal flow sensors and a Pt100 resistance temperature sensor (TetraTec Instruments) that measures the temperature of the gas. The sensors are installed in the insulated plexiglass pipe with a nominal internal diameter of $24 \mathrm{~mm}$. The thermal flow sensors are positioned in the same cross-sectional plane of the pipe and connected to the measurement circuit. One of the thermal flow sensors has a circular cross-section with a diameter of $2.0 \mathrm{~mm}$ and the other thermal flow sensor has a square crosssection with a side length of $2.6 \mathrm{~mm}$.

The thermal flow sensor with the circular crosssection was selected because this shape is the most common in commercially available thermal dispersion mass flow meters, and the thermal flow sensor with the square cross-section was selected because, in combination with the sensor with the circular crosssection, a relatively large difference in the mass flow readings could be expected if the improper measurement characteristics are employed (see Section 1). Keep in mind that the characteristic length of the sensor with the square-cross section is $30 \%$ larger than the characteristic length of the sensor with the circular cross-section, which results in different Reynolds numbers at a given mass flow rate and may also affect the relative difference in the mass flow readings.

Besides the examples presented in Fig. 4, the sensors with other geometries may possibly, if appropriately combined, cause even larger relative differences in the mass flow readings. Therefore, in order to optimize the sensors' geometries, further experimental or numerical investigations could be performed.
The gas-temperature sensor is positioned upstream of the thermal flow sensors and connected to a measurement transmitter (PICO Technologies, PT-104). The control of the developed thermal dispersion mass flow meter and the processing of the measurement signals are realized with a LabVIEWbased program (National Instruments, Ver. 12.0.1).

The measurement circuit for one of the thermal flow sensors is schematically presented in Fig. 6. The thermal flow sensor is connected to a Wheatstone bridge. A programmable DC power supply (National Instruments, PXI-4110) is used to generate the bridge's supply voltage $U_{i}$. A DAQ board (National Instruments, USB-6341) is used to measure both the bridge's output voltage $U_{o}$ and the voltage drop $U_{R_{1}}$ over the resistor $R_{1}$. The electrical resistance of the sensing element within the thermal flow sensor is calculated as:

$$
R=\bar{R} \frac{1+2 U_{o} / U_{i}}{1-2 U_{o} / U_{i}}
$$

where $\bar{R}=\left(R_{1}+R_{2}+R_{3}\right) / 3$ is the average electrical resistance of three thermally stable resistors in a Wheatstone bridge. The temperature of the sensing element is determined from the standard relationship between the electrical resistance and the temperature [20]:

$$
R=R_{0}\left(1+\mathrm{A} T+\mathrm{B} T^{2}\right)
$$

where $\mathrm{A}=3.9083 \times 10^{-3}{ }^{\circ} \mathrm{C}^{-1}$ and $\mathrm{B}=-5.775 \times 10^{-7}{ }^{\circ} \mathrm{C}^{-2}$ are constant values and $R_{0}=100 \Omega$ is the nominal electrical resistance at $0{ }^{\circ} \mathrm{C}$ for Pt100 temperature sensors. The electrical heating power is $P=R I^{2}$, where the electrical current passing through the sensing element is calculated as $I=U_{R_{1}} / R_{1}$. The 
measurement circuit for the other thermal flow sensor is the same as the one presented in Fig. 6, where both Wheatstone bridges are connected to the same DC power supply and DAQ board.

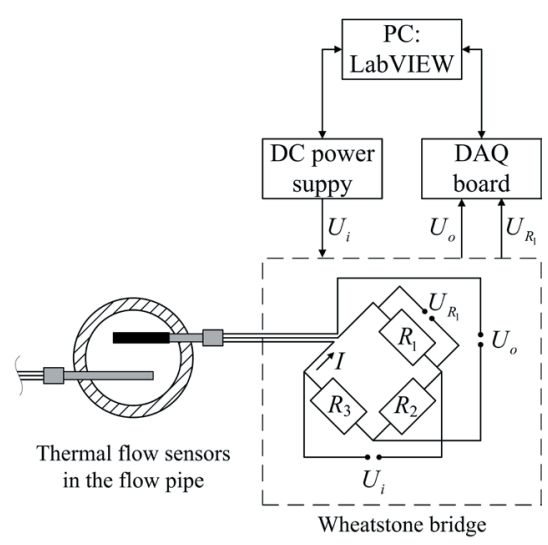

Fig. 6. Scheme of the measurement circuit for the thermal flow sensor

Both thermal flow sensors were calibrated (in non-heated mode; $I=1 \mathrm{~mA}$ ) in the temperature range from 15 to $45{ }^{\circ} \mathrm{C}$, with the reference temperature having an expanded measurement uncertainty of $0.02{ }^{\circ} \mathrm{C}$. The electrical resistances of the thermally stable resistors in both Wheatstone bridges were measured with the expanded measurement uncertainty of a reference value of $0.5 \mathrm{~m} \Omega$. The results presented in the following sections were obtained under steady flow conditions. When all the resistance temperature sensors in the developed thermal dispersion mass flow meter were used to measure the temperature of the gas, the temperature differences between them were less than $0.025^{\circ} \mathrm{C}$.

\subsection{Calibration of the Thermal Dispersion Mass Flow Meter}

The thermal dispersion mass flow meter was calibrated for the following gases: air, oxygen, nitrous oxide, carbon dioxide and argon. The reference mass flow rates in the range from 100 to $350 \mathrm{~g} / \mathrm{min}$ were set and measured by the reference mass flow measurement system with critical flow Venturi nozzles. The temperature difference between each of the thermal flow sensors and the gas was maintained at $10 \mathrm{~K}$. The output signal $P / \Delta T$ (an average value over a time period of $30 \mathrm{~s}$ ) was measured and recorded five times for each mass flow rate. The measurement characteristics of the thermal flow sensors are presented in Figs. 7 and 8. The results were fitted with the Levenberg-Marquardt method using the following form of the measurement model:

$$
\frac{P}{\Delta T}=\frac{1}{c_{1}+\frac{1}{c_{2}+c_{3} \dot{m}^{c_{4}}}}
$$

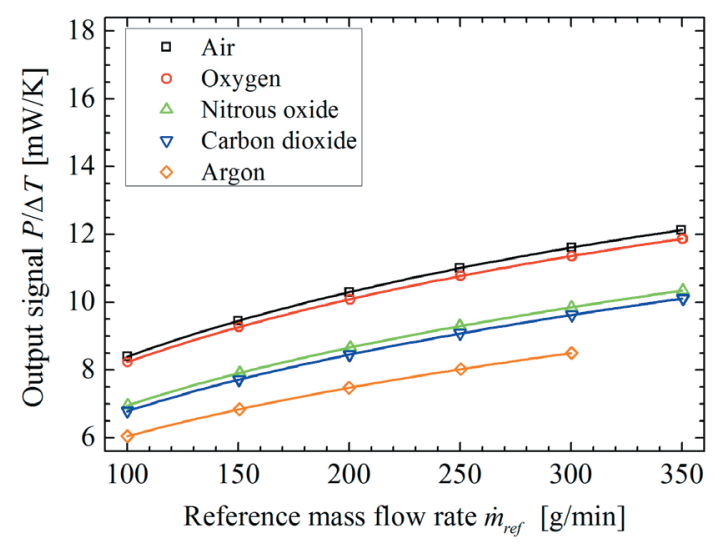

Fig. 7. Measurement characteristics of the thermal flow sensor with the circular cross-section

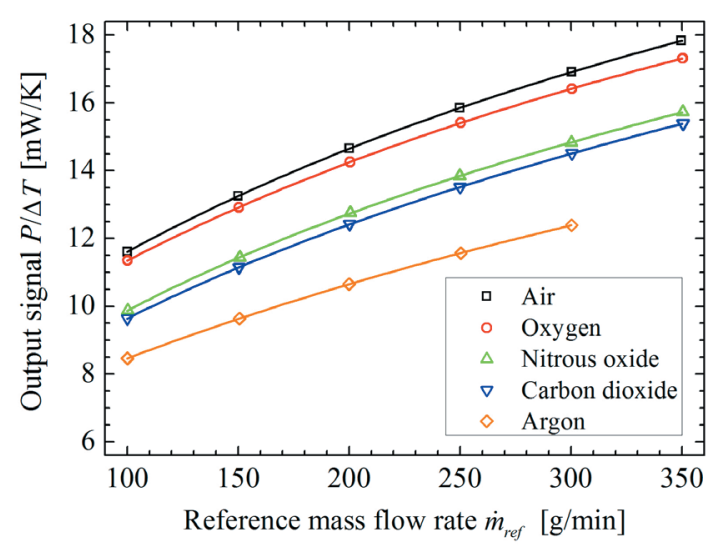

Fig. 8. Measurement characteristics of the thermal flow sensor with the square cross-section

Table 1. Calibration constants and the standard errors of estimates for the measurement model (14) with $\mathrm{P} / \Delta T[\mathrm{~mW} / \mathrm{K}]$ and $\dot{m}[\mathrm{~g} / \mathrm{min}]$

\begin{tabular}{lccccc}
\hline & \multicolumn{5}{l}{ Thermal flow sensor with the circular cross-section } \\
\cline { 2 - 6 } & $c_{1} \times 10^{3}$ & $c_{2}$ & $c_{3} \times 10^{3}$ & $c_{4}$ & $S E E \times 10^{3}$ \\
\hline Air & 46.80 & 6.153 & 162.2 & 0.838 & 3.91 \\
\hline Oxygen & 44.30 & 5.417 & 225.3 & 0.763 & 5.49 \\
\hline Nitrous oxide & 20.67 & 1.664 & 773.7 & 0.461 & 3.33 \\
\hline Carbon dioxide & 19.62 & 1.730 & 726.7 & 0.462 & 2.08 \\
\hline Argon & 27.55 & 2.385 & 42.31 & 0.530 & 1.55 \\
\hline \multicolumn{4}{c}{ Thermal flow sensor with the square cross-section } \\
\cline { 2 - 6 } & $c_{1} \times 10^{3}$ & $c_{2}$ & $c_{3} \times 10^{3}$ & $C_{4}$ & $S E E \times 10^{3}$ \\
\hline Air & 29.49 & 8.945 & 108.6 & 0.952 & 4.74 \\
\hline Oxygen & 30.59 & 9.051 & 99.50 & 0.961 & 7.28 \\
\hline Nitrous oxide & 23.58 & 4.832 & 272.6 & 0.735 & 12.3 \\
\hline Carbon dioxide & 24.24 & 5.335 & 200.4 & 0.779 & 6.05 \\
\hline Argon & 36.38 & 7.053 & 52.18 & 0.998 & 4.00 \\
\hline
\end{tabular}


The values of the calibration constants $c_{1}, c_{2}, c_{3}$ and $c_{4}$ and the standard errors of estimates $S E E$ are presented in Table 1. The thermal flow sensor with the square cross-section has steeper characteristics than the thermal flow sensor with the circular crosssection. This is probably associated with the value of the parameter $c_{4}$, which can be related to the parameter $n$ in the power model for convective heat transfer (Eq. (2)); it is generally expected to be greater for the sensor with the square cross-section [14] and [17]. The sensor with the square cross-section also produces larger values of the output signal, which is a consequence of a more intensive heat transfer.

\subsection{Validation of the Gas-Identification Method}

On the basis of the obtained measurement characteristics, the relative differences in the mass flow readings, $\varepsilon=\left(\dot{m}_{2} / \dot{m}_{1}\right)-1$, can be evaluated. These relative differences are presented in Fig. 9 (with lines) for the defined set of gases, where the actual gas is air. If the measurement characteristics are selected properly (in this case for air), then $\varepsilon=0$, but otherwise $|\varepsilon|>0$. If these experimental results are compared to the results of the theoretical model (see Fig. 3), it is evident that $\varepsilon>0$ for oxygen and $\varepsilon<0$ for nitrous oxide, carbon dioxide and argon in both cases. The experimental results show that $|\varepsilon|$ is greater for nitrous oxide than argon, which contradicts the results of the simple theoretical model. A possible reason for this contradiction could be the limited capability of the one-dimensional mathematical model to describe the actual effects of the gas flow around the thermal flow sensors that are inserted only to certain depths into the flow pipe. These flow conditions also differ, to some extent, from the conditions in which common models for convective heat transfer in the cross-flow were obtained (an example is presented in [21]). In addition to the convective heat transfer that is taken into account in the mathematical model, the radiation heat transfer and the conductive heat transfer in the axial direction [3] affect the performance of the thermal flow sensor as well. Besides, the combined effect of the uncertainties of the gases' thermodynamic and transport properties on the expanded uncertainty of the theoretically determined $\varepsilon$ is up to $4.5 \%$ for nitrous oxide and up to $1.4 \%$ for argon, for the values presented in Fig. 3 (the uncertainties of the gases' properties are given in the NIST REFPROP database [16], and the uncertainty of $\varepsilon$ is calculated in accordance with [19]).

The experimental validation of the gasidentification method was performed at a reference mass flow rate of about $225 \mathrm{~g} / \mathrm{min}$ of air. The results of the validation experiment, presented in Fig. 9 (with symbols) and in Table 2, show that $|\varepsilon|>13 \%$ for nitrous oxide, carbon dioxide and argon, while $|\varepsilon|$ for oxygen and air is 0.94 and $0.06 \%$, respectively. The absolute value of the relative difference in the mass flow readings $|\varepsilon|$ can be defined as the objective function that has to be minimized in order to identify the type of gas. In the given case, air would be properly identified as the actual gas.

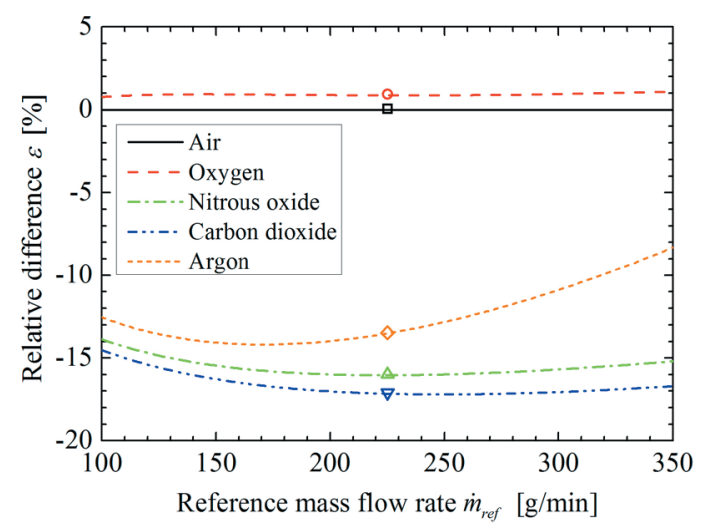

Fig. 9. Relative differences in the mass flow readings for different gases; the actual gas is air; the results of the validation experiment are presented with symbols

Table 2. Results of the validation experiment for the gasidentification method at a reference mass flow rate of $225.10 \mathrm{~g} / \mathrm{min}$ of air

\begin{tabular}{lccccc}
\hline & $\begin{array}{c}\dot{m}_{1} \\
{[\mathrm{~g} / \mathrm{min}]}\end{array}$ & $\begin{array}{c}\dot{m}_{2} \\
{[\mathrm{~g} / \mathrm{min}]}\end{array}$ & $\begin{array}{c}\varepsilon \\
{[\%]}\end{array}$ & $E_{n}$ & $\begin{array}{c}\text { Identified as the } \\
\text { improper gas due } \\
\text { to }\left|E_{n}\right|>1\end{array}$ \\
\hline Air & 224.22 & 224.36 & 0.06 & 0.04 & $\mathrm{o}$ \\
\hline Oxygen & 241.01 & 243.28 & 0.94 & 0.60 & $\mathrm{o}$ \\
\hline $\begin{array}{l}\text { Nitrous } \\
\text { oxide }\end{array}$ & 384.67 & 323.16 & -16.0 & -11.1 & $\checkmark$ \\
\hline $\begin{array}{l}\text { Carbon } \\
\text { dioxide }\end{array}$ & 413.01 & 342.36 & -17.1 & -12.0 & $\checkmark$ \\
\hline Argon & 610.27 & 528.02 & -13.5 & -9.27 & $\checkmark$ \\
\hline
\end{tabular}

However, the question is, whether the proper gas is identified with a sufficient degree of confidence. For this purpose it is reasonable to define a criterion that accounts for the dispersion that could be reasonably attributed to the difference in the mass flow readings, e.g., the normalized error [22]:

$$
E_{n}=\frac{\dot{m}_{2}-\dot{m}_{1}}{\sqrt{U^{2}\left(\dot{m}_{2}\right)+U^{2}\left(\dot{m}_{1}\right)}},
$$

where $U\left(\dot{m}_{1}\right)$ and $U\left(\dot{m}_{2}\right)$ are the expanded measurement uncertainties of the mass flow readings. 
If $\left|E_{n}\right| \leq 1$, the difference in the mass flow readings is statistically insignificant, and if $\left|E_{n}\right|>1$, the difference in the mass flow readings is statistically significant. If the difference in the mass flow readings is statistically significant for a particular gas, this gas can be identified as improper. The difference in the mass flow readings must be statistically significant for all but one gas from the defined set of gases in order to identify the proper gas with a sufficient degree of confidence.

For the evaluation of the measurement uncertainties in Eq. (15), the sources affecting the dispersion of the estimated difference in the mass flow readings should be taken into account. The combined expanded measurement uncertainty of each mass flow reading is estimated not to exceed $1.1 \%$. This estimation takes into account the following contributions: the measurement uncertainty of the reference temperature in the temperature calibration of the thermal flow sensors, the stability of both the electrical heating power and the maintained temperature difference for each of the thermal flow sensors, and the standard errors of estimates of the fitted measurement characteristics. The measurement uncertainty of the reference mass flow rate is not taken into account, because it does not affect the difference between simultaneous measurements by both thermal flow sensors.

The calculated normalized errors for the given validation experiment are presented in Table 2 . Nitrous oxide, carbon dioxide and argon can be identified as the improper gases since $\left|E_{n}\right|>1$ (marked with " $\checkmark$ "). The difference in the mass flow readings is statistically insignificant (marked with "o") for air, which means that air is correctly identified as the proper gas. However, $\left|E_{n}\right| \leq 1$ holds also for oxygen, but it is not the actual gas in the given validation experiment. In order to achieve a statistical significance for all but one gas from the defined set of gases, the developed thermal dispersion mass flow meter could be improved in terms of decreased measurement uncertainties of the mass flow readings or an increased difference in the mass flow readings. The latter can be achieved, for example, by modifying the constructional parameters of the thermal flow sensors in such a way that the difference between the values of the parameters $n_{1}$ and $n_{2}$ is increased.

\section{CONCLUSIONS}

The presented measurement method allows us to identify the type of gas flowing through a thermal dispersion mass flow meter. The gas-identification method can be performed simultaneously with the measurement of the mass flow rate. It represents a novel advancement in the field of thermal mass flow meters. In the first part of the paper the basic principle and physical background of the gas-identification method are discussed. In the second part of the paper the experimental validation of the gas-identification method is presented. Here, we summarize the main findings of the performed research work:

- If a thermal dispersion mass flow meter contains two different thermal flow sensors and the measurement characteristics for the improper gas are employed, the mass flow readings will generally differ. The difference in the mass flow readings was studied by employing a simple, one-dimensional, mathematical model of a thermal flow sensor. The difference depends on the exponents $n_{1}$ and $n_{2}$ in the power model for convective heat transfer, which can be affected by the constructional parameters of the thermal flow sensors, and on the thermodynamic and transport properties of the gas, which can be affected by the operational parameters of the thermal flow sensors.

- For a practical realization of the gas-identification method, two thermal flow sensors with different constructional or operational parameters are required. The mass flow readings of the thermal flow sensors are determined for each gas from a defined set of gases. The identified gas is the one that minimizes the defined objective function, e.g., the absolute value of the relative difference in the mass flow readings. The difference in the mass flow readings should be statistically significant for all but one gas from the defined set of gases in order to identify the proper gas with a sufficient degree of confidence.

- A thermal dispersion mass flow meter containing two thermal flow sensors with circular and square cross-sections was developed and calibrated for the following gases: air, oxygen, nitrous oxide, carbon dioxide and argon. After this, the validation experiment for the gas-identification method was performed for the same set of gases. The minimum absolute value of the relative difference in the mass flow readings was obtained for air, which was the actual gas flowing through the developed thermal dispersion mass flow meter in this case. Air was also correctly identified by employing the normalized error as the objective function. However, the difference in the mass flow readings was found to be statistically insignificant also for oxygen. 
The presented thermal dispersion mass flow meter has to be calibrated for the gases that are expected to flow through the meter in order to perform the gasidentification method. Considering this requirement, it can be employed in such practical applications where defined gases with known compositions are expected, for example, flow systems with different technical, pure or medical gases. Some possible specific applications are medical gas supply systems in hospitals, laser systems for materials processing, calibration of gas chromatography systems, etc. The presented thermal dispersion mass flow meter could be used to correctly measure the mass flow rate of different gases (within the declared measurement uncertainty) and also employed for safety reasons, e.g., for the detection of the improper gas in a particular distribution pipe in a medical gas supply system.

The meter with the integrated gas-identification method has to be capable of identifying the proper gas from the defined set of gases with a sufficient degree of confidence. The presented experimental examples show that nitrous oxide, carbon dioxide and argon were distinguished from air with statistical significance, but it was not possible to distinguish between air and oxygen. Nevertheless, these results can be improved on by decreasing the measurement uncertainties of the mass flow readings. For the developed thermal mass flow meter, the expanded measurement uncertainties of the mass flow readings were estimated to be up to $1.1 \%$. The measurement uncertainty can be reduced by a further optimization of the thermal mass flow meter in the sense of decreasing or correcting the influence of the parameters that affect the mass flow reading, e.g., the heat losses along the stem of the thermal flow sensor. If lower expanded measurement uncertainty, e.g., $0.6 \%$ of the reading, was obtained for the presented thermal mass flow meter, even air and oxygen would be distinguished. In addition to decreasing the measurement uncertainty, another possibility for improving the gas-identification capability of the thermal mass flow meter is to optimize its constructional parameters. If two gases have a similar combination of thermodynamic and transport properties, the constructional parameters of the thermal flow sensors have to be sufficiently different in order to achieve a significant difference in the mass flow readings.

In this investigation, the developed thermal dispersion mass flow meter was used under steady flow conditions. The influences of the varying mass flow rates and temperatures of the measured flow on the results of the gas-identification method should be investigated. The presented realization of the thermal dispersion mass flow meter contains two thermal flow sensors and a separate gas-temperature sensor. Another option for a practical implementation would be a configuration where one or more of the thermal flow sensors operates alternately in the function of a thermal flow sensor and in the function of a gastemperature sensor.

\section{ACKNOWLEDGEMENT}

This work was supported in part by the Slovenian Research Agency (ARRS).

\section{NOMENCLATURE}

\begin{tabular}{|c|c|}
\hline A & constant $\left[{ }^{\circ} \mathrm{C}^{-1}\right]$ \\
\hline$A_{p}$ & cross-sectional area of the flow pipe $\left[\mathrm{m}^{2}\right]$ \\
\hline$a, m, n$ & $\begin{array}{l}\text { parameters of the convective heat transfer } \\
\text { model }\end{array}$ \\
\hline B & constant $\left[{ }^{\circ} \mathrm{C}^{-2}\right]$ \\
\hline \multicolumn{2}{|c|}{$c_{1}, c_{2}, c_{3}, c_{4}$ calibration constants } \\
\hline$c_{p}$ & specific heat of the gas $[\mathrm{J} / \mathrm{kgK}]$ \\
\hline$D$ & $\begin{array}{l}\text { external characteristic length, e.g., diameter, } \\
\text { of the thermal flow sensor [m] }\end{array}$ \\
\hline$E_{n}$ & normalized error \\
\hline$h$ & heat transfer coefficient $\left[\mathrm{W} / \mathrm{m}^{2} \mathrm{~K}\right]$ \\
\hline$I$ & $\begin{array}{l}\text { eletrical current passing through the sensing } \\
\text { element }[\mathrm{A}]\end{array}$ \\
\hline$k$ & coverage factor \\
\hline$L$ & length of the sensing element $[\mathrm{m}]$ \\
\hline$\dot{m}$ & mass flow rate $[\mathrm{kg} / \mathrm{s}]$ \\
\hline$N$ & $\begin{array}{l}\text { number of all layers in the thermal flow } \\
\text { sensor }\end{array}$ \\
\hline $\mathrm{Nu}$ & Nusselt number \\
\hline$P$ & electrical heating power [W] \\
\hline$P / \Delta T$ & $\begin{array}{l}\text { output signal of the thermal flow } \\
\text { sensor }[\mathrm{W} / \mathrm{K}]\end{array}$ \\
\hline $\operatorname{Pr}$ & Prandtl number \\
\hline$R$ & $\begin{array}{l}\text { electrical resistance of the sensing element } \\
{[\Omega]}\end{array}$ \\
\hline $\bar{R}$ & $\begin{array}{l}\text { average electrical resistance of resistors in } \\
\text { the Wheatstone bridge }[\Omega]\end{array}$ \\
\hline & nominal electrical resistance at $0{ }^{\circ} \mathrm{C}[\Omega]$ \\
\hline \multicolumn{2}{|c|}{$\begin{array}{c}R_{1}, R_{2}, R_{3} \text { electrical resistances of resistors in the } \\
\text { Wheatstone bridge }[\Omega]\end{array}$} \\
\hline $\operatorname{Re}$ & Reynolds number \\
\hline$r_{j}$ & $\begin{array}{l}\text { outer radius of the } j^{\text {th }} \text { layer in the thermal } \\
\text { flow sensor }[\mathrm{m}]\end{array}$ \\
\hline SEE & standard error of estimate $[\mathrm{W} / \mathrm{K}]$ \\
\hline$T$ & $\begin{array}{l}\text { temperature of the sensing element within } \\
\text { the thermal flow sensor }\left[{ }^{\circ} \mathrm{C}\right]\end{array}$ \\
\hline$T_{g}$ & temperature of the gas $\left[{ }^{\circ} \mathrm{C}\right]$ \\
\hline
\end{tabular}


$T_{s} \quad$ surface temperature of the thermal flow sensor $\left[{ }^{\circ} \mathrm{C}\right]$

$\Delta T \quad$ temperature difference between the sensing element within the thermal flow sensor and the gas $[\mathrm{K}]$

$U \quad$ expanded measurement uncertainty

$U_{i} \quad$ supply voltage [V]

$U_{o} \quad$ output voltage [V]

$U_{R_{1}} \quad$ voltage drop over the resistor $R_{1}[\mathrm{~V}]$

$\bar{V} \quad$ average velocity of the gas $[\mathrm{m} / \mathrm{s}]$

$\varepsilon \quad$ relative difference in the mass flow readings

$\rho \quad$ density of the gas $\left[\mathrm{kg} / \mathrm{m}^{3}\right]$

$\lambda$ thermal conductivity of the gas [W/mK]

$\lambda_{j} \quad$ thermal conductivity of the $j^{\text {th }}$ layer in the thermal flow sensor [W/mK]

$\eta \quad$ dynamic viscosity of the gas [Pa s]

Subscripts

1 thermal flow sensor 1

2 thermal flow sensor 2

Superscripts

(A) $\quad$ gas $A$

(B) $\quad \operatorname{gas} B$

\section{REFERENCES}

[1] Baker, R.C. (2000). Flow Measurement Handbook: Industrial Designs, Operating Principles, Performance, and Applications. Cambridge University Press, New York, DOI:10.1017/CBO9780511471100.

[2] ISO 14511:2001. Measurement of Fluid Flow in Closed Conduits - Thermal Mass Flowmeters. International Standardization Organization. Geneva.

[3] Olin, J.G. (1999). Industrial thermal mass flowmeters, Part 1: Principles of operation. Measurements and Control, vol. 193.

[4] Olin, J.G. (1999). Industrial thermal mass flowmeters, Part 2: Applications. Measurements and Control, vol. 194.

[5] Badarlis, A., Kumar, V., Pfau, A., Kalfas, A. (2011). Novel sensor geometry for liquids serving in dispersion thermal flow meters. SENSOR+TEST Conferences 2011 - SENSOR Proceedings, p. 78-83.

[6] Baker, R.C., Gimson, C. (2001). The effects of manufacturing methods on the precision of insertion and in-line thermal mass flowmeters. Flow Measurement and Instrumentation, vol. 12, no. 2, p. 113-121, DOI:10.1016/S0955-5986(01)00005-X.

[7] Gibson, J. (2003). The Effect of Gas Properties and Installation Effects on Thermal Mass Flowmeters, Report no. 2002/53, TÜV NEL, East Kilbride.

[8] Pape, D., Hencken, K., Schrag, D., Ott, S., Bärlocher, A., Kramer, A. (2010). Coating Diagnostics for Thermal Mass Flowmeters. IEEE SENSORS
Conference Proceedings, p. 2512-2517, DOI:10.1109/ ICSENS.2010.5690438.

[9] Pape, D., Hencken, K. (2012). Dual Sensor Setup for Thermal Mass Flow Sensor Diagnostics. Proceedings of 16. GMA/ITG-Fachtagung Sensoren und Messsysteme, p. 282-290, DOI:10.5162/ sensoren2012/3.1.2.

[10] Lötters, J. (1999). Economical Thermal Mass Flow Sensor Based on Constant Temperature Anemometry. Sensor Proceedings.

[11] Hardy, J.E., Hylton, J.O., McKnight, T.E. (1999). Empirical correlations for thermal flowmeters covering a wide range of thermal-physical properties. Proceedings of the NCSL Workshop and Symposium, p. 735-750.

[12] Popp, O. (2007). Model based calibration and measurement of thermal dispersion gas mass flow meters. SENSOR+TEST Conferences - SENSOR Proceedings, vol. I, p. 205-210.

[13] Rupnik, K., Kutin, J., Bajsić, I. (2013). Thermal mass flow meter and the gas-identification method. SI patent application, no. P-201300425, Slovenian Intellectual Property Office, Ljubljana.

[14] Cengel, Y.A. (2002). Heat Transfer: A Practical Approach, $2^{\text {nd }}$ ed. McGraw-Hill, New York.

[15] Minkina, W. (1999). Theoretical and experimental identification of the temperature sensor unit step response non-linearity during air temperature measurement. Sensors and Actuators A: Physical, vol. 78, no. 2-3, p. 81-87, DOI:10.1016/S09244247(99)00206-X.

[16] Lemmon, E.W., Huber, M.L., McLinden, M.O. (2010). REFPROP: Reference Fluid Thermodynamic and Transport Properties, NIST Standard Reference Database 23, Version 9.0. National Institute of Standards and Technology, Gaithersburg.

[17] Sparrow, E.M., Abraham, J.P., Tong, J.C.K. (2004). Archival correlations for average heat transfer coefficients for non-circular and circular cylinders and for spheres in cross-flow. International Journal of Heat and Mass Transfer, vol. 47, no. 24, p. 5285-5296, DOI:10.1016/j.ijheatmasstransfer.2004.06.024.

[18] ISO 9300:2005. Measurement of Gas Flow by Means of Critical Flow Venturi Nozzles. International Standardization Organization. Geneva.

[19] JCGM 100:2008. Evaluation of Measurement DataGuide to the Expression of Uncertainty in Measurement. Bureau International des Poids et Mesures. Sevres.

[20] IEC 60751:2008. Industrial Platinum Resistance Thermometers and Platinum Temperature Sensors. International Electrotechnical Commission. Geneva.

[21] Sanitjai, S., Goldstein, R.J. (2004). Forced convection heat transfer from a circular cylinder in crossflow to air and liquids. International Journal of Heat and Mass Transfer, vol. 47, p. 4795-4805, DOI:10.1016/j. ijheatmasstransfer.2004.05.012.

[22] ISO 13528:2005. Statistical Methods for Use in Proficiency Testing by Interlaboratory Comparisons. International Standardization Organization, Geneva. 